Research Article

\title{
Z-Control on COVID-19-Exposed Patients in Quarantine
}

\author{
Nita H. Shah $\mathbb{D}$, Nisha Sheoran, and Ekta Jayswal \\ Department of Mathematics, Gujarat University, Ahmedabad, Gujarat, India \\ Correspondence should be addressed to Nita H. Shah; nitahshah@gmail.com
}

Received 3 April 2020; Accepted 23 May 2020; Published 19 June 2020

Academic Editor: Sining Zheng

Copyright $\odot 2020$ Nita H. Shah et al. This is an open access article distributed under the Creative Commons Attribution License, which permits unrestricted use, distribution, and reproduction in any medium, provided the original work is properly cited.

In this paper, a mathematical model for diabetic or hypertensive patients exposed to COVID-19 is formulated along with a set of first-order nonlinear differential equations. The system is said to exhibit two equilibria, namely, exposure-free and endemic points. The reproduction number is obtained for each equilibrium point. Local stability conditions are derived for both equilibria, and global stability is studied for the endemic equilibrium point. This model is investigated along with Z-control in order to eliminate chaos and oscillation epidemiologically showing the importance of quarantine in the COVID-19 environment.

\section{Introduction}

COVID-19, an infectious disease with the first case reported in Wuhan city of China, has spread throughout the world. On 30 January 2020, the WHO declared its outbreak as a "public health emergency of international concern." As on 2 April 2020, 08:02 GMT, it has caused 47,249 deaths worldwide and a total of 936,237 cases have been confirmed [1]. COVID-19 spreads through the contact of individuals with infected persons when they cough or sneeze. It is a respiratory disease with mild to moderate symptoms like dry cough, fever, and tiredness, and in more severe cases difficulty breathing [2]. Also, few individuals showing mild symptoms of the disease may recover themselves if they avoid contacting infected cases and maintain good hygiene. COVID-19 is a major health threat to those with a past medical history and also to those who are older than 60 years (elderly population). This was reported by Li et al. [3], who calculated the median age of 425 patients infected with COVID-19 in Wuhan, China, as 59 years and almost half of the patients were 60 years old.

Governments throughout the world are taking several preventive measures to control the spread of the epidemic. Preventing the spread is the only way since no vaccine has been developed till date to fight the virus. Preventive measures include maintaining at least 1 meter distance from a person sneezing or coughing, washing hands regularly, and maintaining social distance.
Various mathematical models have been developed so far to address various challenges in predicting the spread of COVID-19 disease. Batista [4] has used the basic SIR-model to find the actual size of the epidemic. Peng et al. [5] analysed the scenario of COVID-19 in China by formulating the SEIR dynamical system and have predicted that the situation will be under control at the beginning of April.

Sun et al. [6] discussed various aspects of COVID-19 situation in China which helps understand the fatality rate and transmission rate of COVID-19 and control the epidemic spread. In the case of COVID-19 epidemic, exposure to disease plays a vital role in the spread of the disease. Rabajante [7] studied various models and concluded that with the basic reproduction number being 2 and considering the 14-day infectious period, if an infected person stays for more than 9 hours with others, he could infect others. If the exposure time is 18 hours, the model recommends full protection with more than $70 \%$ effectiveness to the attendees of the social gathering. In order to control the disease, the importance of travel quarantine or travel restriction, which delayed the progression of disease, in Wuhan was studied by Chinazzi et al. [8]. A similar result was also shown by Kucharski et al. [9]. They showed that with the air travel restriction in Wuhan, the daily reproduction number declined from 2.35 to 1.05 . There is also a model evaluated by Tang et al. [10] and Tang et al. [11] in which they divided the subpopulation into quarantined and unquarantined classes to understand the transmission risk of the epidemic. 
Table 1: Parametric definitions and their values.

\begin{tabular}{lccc}
\hline Notations & Description & Parametric values \\
\hline$B$ & Birth rate & 1 & Assumed \\
$\beta_{1}$ & Transmission rate of individuals from susceptible class to exposed class & 0.9 & Calculated \\
$\beta_{2}$ & Force of infection & 0.01 & Assumed \\
$\beta_{3}$ & Rate at which exposed individuals quarantine themselves & 0.80 & Calculated \\
$\beta_{4}$ & Rate at which susceptible individuals quarantine themselves & 0.60 & Calculated \\
$a, b, d$ & Half-saturation constants & $2,10,0.4$ & Assumed \\
$c_{1}, c_{2}$ & Conversion efficiency & 1,2 & Assumed \\
\hline
\end{tabular}

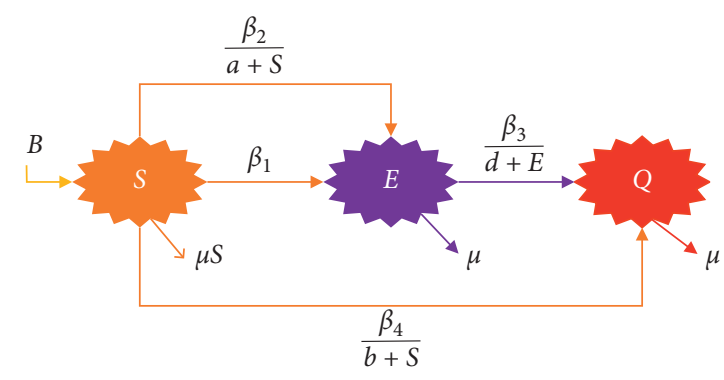

Figure 1: Compartmental diagram of flow of individuals from one compartment to another compartment.

From the studies so far, we have observed that quarantine or isolation plays a huge role in controlling the disease spread. Here, we have developed a model with Z-control applied to the quarantine class to obtain the required exposure state in order to make the system free of chaos. Few basic models of Z-control include prey-predator model by Alzahrani et al. [12] and epidemic model by Samanta [13].

The construction of the paper is as follows: formulation and description of the mathematical model are given in Section 2. In Section 3, the reproduction number is calculated for the state when there is no exposure to COVID-19 and for the endemic state. In Section 4, local stability and global stability are discussed. In Section 5, the model is constructed with Z-controller and $u(t)$ is calculated. In Section 6, a numerical simulation is performed. Finally, the paper concludes with Section 7.

\section{Mathematical Model}

Mathematically, the compartmental model for the individuals already suffering from diabetes or hypertension who are at a higher risk of getting infected with COVID-19 is formulated in this section with three mutually exclusive disjoint classes. The classes are as follows: S: susceptible class, diagnosed with diabetes or hypertension; $E$ : exposed class; and $Q$ : quarantine class.

Notations and parametric values used in the formulation of dynamical system are given in Table 1.
The model considers the susceptible class as a population diagnosed with diabetes or suffering from hypertension. Here, recruitment of new individuals occurs at the rate $B$, and all the individuals in their respective compartments suffer death at the constant rate $\mu$. We have considered the saturated incidence as $\beta_{2} S E /(a+S)$, where $a$ is the saturation constant and $\beta_{2}$ is the force of infection as shown in Figure 1.

Figure 1 gives rise to the following system of nonlinear differential equations:

$$
\begin{aligned}
& \frac{\mathrm{d} S}{\mathrm{~d} t}=B S-\beta_{1} S E-\frac{\beta_{2} S E}{a+S}-\frac{\beta_{4} S Q}{b+S}-\mu S^{2}, \\
& \frac{\mathrm{d} E}{\mathrm{~d} t}=\beta_{1} S E+\frac{\beta_{2} S E}{a+S}-\frac{\beta_{3} E Q}{d+E}-\mu E, \\
& \frac{\mathrm{d} Q}{\mathrm{~d} t}=\frac{c_{1} \beta_{3} E Q}{d+E}+\frac{c_{2} \beta_{4} S Q}{b+S}-\mu Q,
\end{aligned}
$$

with $S>0, E>0, Q>0$.

Equating $\mathrm{d} S / \mathrm{d} t=0, \mathrm{~d} E / \mathrm{d} t=0, \mathrm{~d} Q / \mathrm{d} t=0$ and solving, we get the following equilibrium points:

(i) Exposure-free equilibrium point $E^{1}\left(b \mu /\left(\beta_{4} c_{2}-\mu\right)\right.$, $\left.0, b c_{2}\left(B \beta_{4} c_{2}-b \mu^{2}-B \mu\right) /\left(\beta_{4} c_{2}-\mu\right)^{2}\right)$.

(ii) Endemic equilibrium point $E_{d}^{*}\left(S^{*}, E^{*}, Q^{*}\right)$

$$
\begin{aligned}
& S^{*}=r_{1}, \\
& E^{*}=-\left(\left(d\left(r_{1}\left(\beta_{4} c_{2}-\mu\right)-b \mu\right)\right) /\left(r_{1}\left(\beta_{3} c_{1}+\beta_{4} c_{2}-\mu\right)\right.\right. \\
& \left.\left.+b \beta_{3} c_{1}-b \mu\right)\right),
\end{aligned}
$$




$$
\begin{aligned}
& S^{*}=r_{1} \text {, } \\
& E^{*}=-\frac{d\left(r_{1}\left(\beta_{4} c_{2}-\mu\right)-b \mu\right)}{r_{1}\left(\beta_{3} c_{1}+\beta_{4} c_{2}-\mu\right)+b \beta_{3} c_{1}-b \mu}, \\
& Q^{*}=r_{1}^{2}\left(\beta_{1}\left(a b \beta_{1} c_{1}^{2} \mu\left(c_{1}-c_{2}\right)(b-a)-a c_{1}^{2} \mu^{2}(a-b)+a b \beta_{2} c_{1}^{2} \mu\left(c_{2}-c_{1}\right)\right) \beta_{3}^{2}\right. \\
& +\beta_{1} \beta_{3}\left(a^{2} b \beta_{1} \beta_{4} c_{1} c_{2} \mu\left(c_{2}-c_{1}\right)+a b \beta_{1} c_{1} \mu^{2}\left(2 c_{1}(a-b)-a c_{2}\right)-a c_{1}^{2} c_{2} \beta_{4} \mu(a-b)-a b \beta_{2} \beta_{4} c_{1} c_{2} \mu\left(c_{1}-c_{2}\right)+c_{1} \mu^{2}\left(b^{3} \beta_{1} c_{2}+2 a^{2} c_{1} \mu\right)\right. \\
& \left.+a b c_{1} \beta_{2} \mu^{2}\left(2 c_{1}-c_{2} \beta_{2}\right)-a b c_{1} \mu^{3}\left(2 c_{1}-c_{2}\right)-b^{2} c_{1} c_{2} \mu^{2}\left(\beta_{2}-\mu\right)\right) \\
& +\beta_{1}\left(\beta _ { 4 } \left(a^{2}\left(c_{1} c_{2} \beta_{4} \mu^{2}\left(2 \mu-c_{2} \beta_{4}\right)-c_{1} \mu^{2}\left(\mu^{2}-2 \beta_{4} c_{2} \mu-b \beta_{1} \beta_{4} c_{2}\right)\right)+a\left(b c_{2}^{2} \beta_{4} \mu^{2}\left(c_{2} \beta_{4}-\beta_{1} b\right)+b^{2} \beta_{1} \mu^{3}\left(c_{1}+c_{2}\right)\right.\right.\right. \\
& \left.\left.+b \beta_{4} c_{2} \mu^{2}\left(\beta_{2} c_{1}-c_{1} \mu-2 c_{2} \mu\right)-b \mu^{3}\left(\beta_{2} c_{1}-c_{1} \mu-c_{2} \mu\right)\right)-b^{2} c_{2} \mu^{2}\left(b \beta_{1} \mu+c_{2} \beta_{2} \beta_{4}-\mu\left(c_{2} \beta_{4}+\beta_{2}-\mu\right)\right)\right) \\
& +r_{1}\left(\beta _ { 3 } \beta _ { 4 } \left(\beta _ { 3 } \left(\left(c_{1}-c_{2}\right) a b c_{1}^{2}\left(b \beta_{1} \mu(b-a)+B \beta_{1}(a+b)+B \beta_{2}\right)+\left(\beta_{2}+\mu\right) a b c_{1}^{2} \mu\left(b-c_{1}\right)\right.\right.\right. \\
& \left.+B a c_{1}^{3} \mu(a-b)\right)+\left(c_{1}-c_{2}\right)\left(a^{2} b c_{1} \beta_{1}\left(2 \mu-c_{2} \beta_{4}\right)(b \mu-B)+B a b c_{1} c_{2} \beta_{4}\left(\beta_{2}-\mu\right)-a b^{2} \beta_{1} c_{1} \mu\left(2 b \mu-\beta_{1} d\right)\right. \\
& \left.+b^{2} c_{1} \mu\left(b \beta_{1}^{3} d+2 a \beta_{2} \mu\right)\right)-a b c_{1} \mu^{2}\left(2 c_{1}-c_{2}\right)\left(b \beta_{4} c_{2}+\mu\right)-\left(a \mu+b \beta_{2}\right) c_{1}^{2} \mu\left(b \beta_{1} d+2 B a\right) \\
& -\left(\beta_{2} c_{1}-\beta_{2} c_{2}-c_{1} \mu\right) a b^{2} \beta_{4} c_{1} c_{2} \mu+2 B a c_{1}^{2} \mu\left(\beta_{4} a c_{2}+b^{2}\right)+b^{2} \beta_{1} c_{1} d \mu\left(\beta_{2}+c_{1} \mu\right)+B b^{2} \mu c_{1} c_{2}\left(\beta_{2}-\mu\right) \\
& \left.+B a b c_{1} \mu\left(\beta_{2} c_{2}+2 c_{1} \mu+c_{2} \mu\right)\right)+\beta_{4}\left(( c _ { 1 } - c _ { 2 } ) \left(\left(\beta_{2}-\mu\right)\left(b^{2} \beta_{1} d \mu\left(\mu-\beta_{4}\right)-a b^{2} \mu^{2}\left(\mu-c_{2} \beta_{4}\right)\right)\right.\right. \\
& \left.+a b \beta_{1} \mu^{2}\left(b \mu(b-a)+d\left(b \beta_{1}+\mu\right)\right)-b \mu^{2}\left(b^{2} d \beta_{1}^{2}+a^{2} \mu^{2}\right)+\left(a \mu-\beta_{1} d\right)\left(b \beta_{1}-\beta_{4} c_{2}+2 \mu\right) a b \beta_{4} c_{2} \mu\right) \\
& +B a \beta_{4} c_{2} \mu\left(b \beta_{1}-\beta_{4} c_{2}\right)\left(b c_{2}-a c_{1}\right)-\left(c_{1}+c_{2}\right) B a b \mu^{2}\left(b \beta_{1}+\mu\right)+B a c_{1} \mu^{2}\left(a b-2 a \beta_{4} c_{2}\right) \\
& \left.+\left(\beta_{2}-\mu\right) B b c_{2} \mu\left(b \mu+\beta_{4} c_{2}-a \beta_{4} c_{1}\right)+B \mu^{2}\left(b c_{2}\left(2 \beta_{4} c_{2}+b \beta_{1}\right)+a c_{1}\left(a \mu+b \beta_{2}\right)\right)\right)+\beta_{3} \beta_{4}\left(\beta_{3}\left(c_{1}-c_{2}\right) B a b^{2} c_{1}^{2}\left(\beta_{1}(a-b)+\beta_{2}\right)\right. \\
& +\left(c_{1}-c_{2}\right)\left(a^{2} b^{2} B \beta_{1} c_{1}\left(\beta_{4} c_{2}-\mu\right)+a^{2} b \beta_{1} c_{1} d \mu\left(c_{2} \beta_{4}-b \beta_{1}\right)+a b \beta_{4} c_{1} c_{2} \mu\left(2 B a-b \beta_{1} d\right)+a b^{3} \beta_{1} c_{1} \mu\left(\beta_{1} d+2 B\right)\right. \\
& \left.+a b^{2} \beta_{2} c_{1}\left(B \beta_{4} c_{2}-2 \beta_{1} d \mu\right)+a b \beta_{2} c_{1} \mu\left(\beta_{4} c_{2} d-2 B b\right)+b^{3} \beta_{1} c_{1} d \mu\left(\beta_{2}-\mu\right)\right)-a b c_{1}^{2} d \mu\left(B b \beta_{4} c_{2}+2 a \beta_{1} \mu\right)+a^{2} c_{1} c_{2} d \mu^{2}\left(b \beta_{1}+\beta_{4} c_{1}\right) \\
& +a b^{2} \beta_{1} c_{1} d \mu^{2}\left(3 c_{1}-2 c_{2}\right)+\left(2 c_{1}-c_{2}\right) a b c_{1} \mu^{2}\left(B(a-b)+d \beta_{2}\right)-c_{1}^{2} d \mu^{3}\left(a^{2}+b^{2}\right)+b c_{1}^{2} d \mu\left(2 a \mu-b \beta_{2}^{2}\right)+b^{2} \beta_{2} c_{1} c_{2} d \mu\left(\beta_{2}-\mu\right) \\
& \left.+b c_{1}^{2} d \mu^{2}\left(2 b \beta_{2}-\beta_{4} c_{2}\right)\right)+\beta_{4}\left(\beta_{4} b\left(\beta_{4}\left(a c_{2}^{2} \mu\left(\left(c_{1}-c_{2}\right) b\left(a \beta_{1}+\beta_{2}\right) d+B a\right)+c_{1} d \mu(a-b)\right)\right)\right. \\
& +\left(c_{1}-c_{2}\right) b \mu c_{2}\left(b a^{2} \beta_{1}\left(\beta_{1} d+B\right)-2 a b \beta_{2}\left(b \beta_{1} d+a \mu B\right)-B a b\left(\beta_{2}-\mu\right)-b \beta_{2}^{2} d\right)+c_{2} d \mu^{2}\left(b^{2}\left(2 c_{1}-c_{2}\right)\left(a \beta_{1}+\beta_{2}\right)-a^{2} b \beta_{1}\left(3 c_{1}-2 c_{2}\right)\right. \\
& \left.-a c_{1}\left(2 a \mu+3 b \beta_{2}\right)+a b\left(2 \beta_{2} c_{2}+3 c_{1} \mu\right)-b^{2} c_{1} \mu\right)+b\left(( c _ { 1 } - c _ { 2 } ) b \left(d \mu^{3} \beta_{1}\left(1-b^{2}\right)\right.\right. \\
& \left.+\beta_{2} b(2 a-b)+b^{2} c_{1}\right)+\left(2 c_{1}-c_{2}\right) a b d \mu^{3}\left(a \beta_{1}+\beta_{2}\right)-d \mu^{2}\left(\left(3 c_{1}-c_{2}\right) a b^{2} \beta_{1} \mu-\left(a^{2}+b^{2}\right) c_{1}\right. \\
& \left.\left.\left.+\left(2 a-b \beta_{2}^{2}\right) b c_{1}+b^{2} \beta_{2}\left(\beta_{2} c_{2}+2 \mu\right)+\mu b^{2}\left(\beta_{2} c_{2}+\beta_{1} c_{1}\right)\right)\right)\right) \cdot\left(\left(\left(c_{1} \mu\left(c_{1}-c_{2} \mu\right) \beta_{3}^{2}+\mu^{2}\left(c_{2}-c_{1} \mu\right) \beta_{3}+\mu^{3}\right) b^{3} \beta_{1}\right.\right. \\
& +\left(\left(c_{1}^{2} \mu^{2}-a c_{1} \beta_{1} \beta_{4}\left(c_{1}-c_{2}\right)^{2}+c_{1} \beta_{2} \mu\left(c_{2}-c_{1}\right) \beta_{3}^{2}+\left(\left(\left(2 a \beta_{4} \mu\left(c_{1}-c_{2}\right)^{2}+2 a \mu^{2}\left(2 c_{1}-c_{2}\right)\right) \beta_{1}\right.\right.\right.\right. \\
& \left.+\beta_{2} \mu^{2}\left(2 c_{1}-c_{2}\right)+\beta_{4} c_{1} c_{2} \mu\left(\mu-\beta_{2}\right)+\beta_{2} \beta_{4} c_{2}^{2} \mu-2 c_{1} \mu^{3}\right) \beta_{3}-\left(a \mu^{2}\left(c_{1} \beta_{4}-2\left(c_{2}+\mu\right)\right) \beta_{1}\right. \\
& \left.-\left(\beta_{4} c_{2} \mu^{2}-\mu^{3}\right)\left(\mu-\beta_{2}\right)\right) b^{2}+\left(\left(\left(c_{1}\left(c_{1}-c_{2}\right) a^{2}\left(\beta_{4}\left(c_{1}-c_{2}\right)+\mu\right) \beta_{1}+a c_{1} \beta_{2} \beta_{4}\left(c_{2}^{2}+c_{1}^{2}\right)-2 a c_{1}^{2}\left(\beta_{2} \beta_{4} c_{2}+\mu^{2}\right)\right.\right.\right. \\
& \left.-a c_{1} \mu\left(\beta_{2} c_{2}+\beta_{4} c_{1}^{2}-\beta_{2} c_{1}\right)\right) \beta_{3}^{2}+\left(\left(\left(c_{1}-c_{2}\right)^{2} a^{2} \beta_{4}\left(c_{1} c_{2} \beta_{4}-2 \mu\right)-a^{2} \mu^{2}\left(2 c_{1}-c_{2}\right)\right) \beta_{1}\right. \\
& +2 a c_{1} \mu^{2}\left(2 \mu+c_{1} \beta_{4}-\beta_{2}\right)+a c_{2} \beta_{2}\left(c_{2}^{2} \beta_{4}^{2}+\mu^{2}\right)+2 a c_{2} \beta_{2} \beta_{4} \mu\left(2 c_{1}-c_{2}\right)+a \beta_{4}^{2} c_{1} c_{2}^{2}\left(\mu-2 \beta_{2}\right) \\
& \left.-a c_{1}^{2} c_{2} \beta_{4}^{2}\left(\mu-\beta_{2}\right)-a c_{1} \beta_{4} \mu\left(5 c_{2} \mu+2 c_{1} \beta_{2}\right)\right) \beta_{3}+\left(a^{2} c_{2} \beta_{4}^{2} \mu\left(c_{2}-c_{1}\right)+a^{2} \mu^{2}\left(c_{1} \beta_{4}-2 c_{2} \beta_{4}+\mu\right) \beta_{1}\right. \\
& \left.+a \beta_{4} \mu\left(c_{1} \mu\left(\beta_{2}+c_{2} \beta_{4}\right)-c_{2} \beta_{2}\right)-a \mu\left(\mu^{2}\left(2 \mu+c_{1} \beta_{4}\right)+\beta_{2}\left(c_{1} c_{2} \beta_{4}^{2}-\mu^{2}\right)\right)-2 a c_{2} \mu^{2} \beta_{4}\left(c_{2} \beta_{4}-2 \mu\right)\right) b \\
& +\left(a^{2} \beta_{4} c_{1}^{2} \mu\left(c_{1}-c_{2}\right)+a^{2} c_{1}^{2} \mu^{2}\right) \beta_{3}^{2}+\left(2 a^{2} c_{1} c_{2} \beta_{4}^{2} \mu\left(c_{1}-c_{2}\right)-2 a^{2} c_{1} \mu^{2}\left(c_{1} \beta_{4}-2 c_{2} \beta_{4}-\mu\right)\right) \beta_{3} \\
& \left.\left.+a^{2} \mu^{2} \beta_{4}\left(\left(3 c_{2}+c_{1}\right)-3 a^{2} \beta_{4} c_{2} \mu^{3}+a^{2} \beta_{4}^{3} c_{2} \mu\left(c_{1} c_{2}-2 c_{1} \mu-c_{2}^{2}\right)+a^{2} \mu^{4}\right)\right)\right)^{-1},
\end{aligned}
$$


where

$$
\begin{aligned}
r_{1}= & \operatorname{Root} \text { of }\left(\left(\beta_{3} c_{1}+\beta_{4} c_{2}-\mu\right) Z^{3}\right. \\
& +\left((a \mu-B)\left(\beta_{3} c_{1}+\beta_{4} c_{2}-\mu\right)+b \mu\left(\beta_{3} c_{1}+\mu\right)+\beta_{1} d\left(\beta_{4}\left(c_{1}-c_{2}\right)+\mu\right)\right) Z^{2} \\
& +\left(\left(\beta_{3} c_{1}-\mu\right)(a b \mu-B b)-B a\left(\beta_{3} c_{1}+\beta_{4} c_{2}-\mu\right)+\left(a \beta_{1} d+\beta_{2} d\right)\left(\beta_{4}\left(c_{1}-c_{2}\right)+\mu\right)+d \mu\left(b \beta_{1}-\beta_{4} c_{1}\right)\right) Z \\
& \left.+a b B\left(\beta_{3} c_{1}+\beta_{4} c_{1}-\mu\right)+b d \mu\left(a \beta_{1}+\beta_{2}\right)\right)
\end{aligned}
$$

\section{Reproduction Number}

$\mathrm{n}$ this paper, the reproduction number is defined as the number of secondary individuals who are asked to go for quarantine or social distancing due to a single quarantined susceptible individual exposed to COVID-19.
For this model, the reproduction number for exposurefree equilibrium and that for endemic stage equilibrium are calculated. Using the next-generation matrix method by Diekmann et al. [14], we get the following Jacobian matrices:

$$
\begin{aligned}
& F=\left[\begin{array}{ccc}
\frac{\beta_{2} S}{a+S}+\beta_{1} S & 0 & \beta_{1} E+\frac{\beta_{2} E}{a+S}-\frac{\beta_{2} S E}{(a+S)^{2}} \\
\frac{c_{1} \beta_{3} Q}{d+E}-\frac{c_{1} \beta_{3} E Q}{(d+E)^{2}} & \frac{c_{1} \beta_{3} E}{d+E}+\frac{c_{2} \beta_{4} S}{b+S} & \frac{c_{2} \beta_{4} Q}{b+S}-\frac{c_{2} \beta_{4} S Q}{(b+S)^{2}} \\
0 & 0 & 0
\end{array}\right] \\
& V=\left[\begin{array}{ccc}
\frac{\beta_{3} E}{d+E}-\frac{\beta_{3} E Q}{(d+E)^{2}}+\mu \frac{\beta_{3} E}{d+E} & 0 \\
0 & \mu & 0 \\
\frac{\beta_{2} S}{a+S}+\beta_{1} S & \frac{\beta_{4} S}{b+S}-B+2 \mu S+\frac{\beta_{2} E}{a+S}-\frac{\beta_{2} E S}{(a+S)^{2}}+\frac{\beta_{4} Q}{b+S}-\frac{\beta_{4} Q S}{(b+S)^{2}}+\beta_{1} E
\end{array}\right]
\end{aligned}
$$

Reproduction numbers $R_{0 E^{1}}$ and $R_{0 E_{d}^{*}}$ are defined as the spectral radius of $F V^{-1}$ at $E^{1}$ and $E_{d}^{*}$, respectively, which are numerically computed as 2.66 and 5.35 .

\section{Stability Analysis}

In this section, local stability and global stability for the equilibrium points are studied.

4.1. Local Stability. Local stability is obtained for all of its equilibrium points by using the Routh-Hurwitz criterion [15].

Theorem 1. Exposure-free equilibrium point $E^{1}$ is said to be locally asymptotically stable if it satisfies $\left(\beta_{4} c_{2}-\mu\right)\left(\beta_{1} \mu d+\right.$ $\left.c_{2} \beta_{3} B\right)<\left[\mu+\left(b \beta_{2} \mu /\left(a\left(\beta_{4} c_{2}-\mu\right)+b \mu\right)\right)\right]\left(\left(d\left(\beta_{4} c_{2}-\mu\right)^{2}\right) / b\right)$ $+c_{2} \beta_{3} b \mu^{2}$ and $\mu(b \mu+B)<c_{2} B \beta_{4}$.

Proof. The elements of the Jacobian matrix of system (1) at $E^{1}$ are

$$
\begin{aligned}
& a_{11}=B-\frac{\left(B c_{2} \beta_{4}-b \mu^{2}-B \mu\right)}{\beta_{4} c_{2}}-\frac{2 \mu^{2} b}{\beta_{4} c_{2}-\mu}, \\
& a_{12}=-\frac{\beta_{2} b \mu}{\left(a\left(\beta_{4} c_{2}-\mu\right)+b \mu\right)}-\frac{\beta_{1} b \mu}{\beta_{4} c_{2}-\mu}, \\
& a_{13}=-\frac{\mu}{c_{2}}, \\
& a_{21}=0,
\end{aligned}
$$$$
a_{22}=\frac{b \beta_{1} \mu}{\beta_{4} c_{2}-\mu}-\frac{b \beta_{2} \mu}{\left(a\left(\beta_{4} c_{2}-\mu\right)+b \mu\right)}+\frac{b c_{2} \beta_{3}\left(B \beta_{4} c_{2}-b \mu^{2}-B \mu\right)}{d\left(\beta_{4} c_{2}-\mu\right)^{2}}-\mu,
$$$$
a_{23}=0 \text {, }
$$$$
a_{31}=\frac{\left(B c_{2} \beta_{4}-b \mu^{2}-B \mu\right)}{\beta_{4}},
$$$$
a_{32}=\frac{c_{1} c_{2} b \beta_{3}\left(B \beta_{4} c_{2}-b \mu^{2}-B \mu\right)}{d\left(\beta_{4} c_{2}-\mu\right)},
$$

$a_{33}=0$. 
The eigenvalues of this Jacobian matrix are

$$
\begin{aligned}
\lambda_{1} & =a_{22}, \\
\lambda_{2,3} & =\frac{1}{2}\left(-a_{11} \pm \sqrt{a_{11}^{2}-4 a_{13} a_{31}}\right) .
\end{aligned}
$$

These eigenvalues are negative if $a_{22}<0$ and $a_{13} a_{31}<0$, i.e., $\left(\beta_{4} c_{2}-\mu\right)\left(\beta_{1} \mu d+c_{2} \beta_{3} B\right)<\left[\mu+\left(b \beta_{2} \mu /\left(a\left(\beta_{4} c_{2}-\mu\right)+b\right.\right.\right.$ $\mu))]\left(\left(d\left(\beta_{4} c_{2}-\mu\right)^{2}\right) / b\right)+c_{2} \beta_{3} b \mu^{2}$ and $\mu(b \mu+B)<c_{2} B \beta_{4}$. Hence, satisfying the Routh-Hurwitz criterion, the system is locally asymptotically stable.

Theorem 2. Endemic point is said to be locally asymptotically stable if it satisfies

$$
\begin{aligned}
& \text { (1) } B<a f_{3} E^{*}+b f_{5} Q^{*}+\beta_{1} E^{*}+2 \mu S^{*} \\
& \text { (2) } \beta_{1} S^{*}+d f_{6} Q^{*}<\mu+f_{1} S^{*} \\
& \text { (3) } c_{1} f_{4} E^{*}+c_{2} f_{2} S^{*}<\mu \\
& \text { (4) }\left(\beta_{1} S^{*}-f_{1} S^{*}+d f_{6} Q^{*}-\mu\right)\left(c_{1} f_{4} E^{*}+c_{2} f_{2} S^{*}-\mu\right) \\
& >c_{1} d f_{4} f_{6} E^{*} Q^{*} .
\end{aligned}
$$

Proof. Let us consider the following Jacobian matrix of system (1) at $E_{d}^{*}$ :

$$
J^{*}=\left[\begin{array}{ccc}
-b_{11} & -\left(f_{1}+\beta_{1}\right) S^{*} & -S^{*} f_{2} \\
\left(\beta_{1}+a f_{3}\right) E^{*} & -b_{22} & f_{4} E^{*} \\
c_{2} f_{5} b Q^{*} & c_{1} f_{6} d Q^{*} & -b_{33}
\end{array}\right],
$$

where $\quad b_{11}=-B+a f_{3} E^{*}+b f_{5} Q^{*}+\beta_{1} E^{*}+2 \mu S^{*}, \quad b_{22}=$ $-\beta_{1} S^{*}+f_{1} S^{*}-d f_{6} Q^{*}+\mu, \quad b_{33}=-c_{1} f_{4} E^{*}-c_{2} f_{2} S^{*}+\mu$, $f_{1}=\beta_{2} /(a+S), \quad f_{2}=\beta_{4} /(b+S), \quad f_{3}=\beta_{2} /(a+S)^{2}, \quad f_{4}=$ $\beta_{3} /(d+E), f_{5}=\beta_{4} /(b+S)^{2}$, and $f_{6}=\beta_{3} /(d+E)^{2}$.

The characteristic polynomial of (8) is given by

$$
\alpha^{3}+\varepsilon_{2} \alpha^{2}+\varepsilon_{1} \alpha+\varepsilon_{0},
$$

where $\varepsilon_{0}=c_{1} d f_{2} f_{6}\left(a f_{3}+\beta_{1}\right) E^{*} Q^{*} S^{*}+\left(\beta_{1}+f_{1}\right)\left(c_{2} f_{4}\right.$ $\left.f_{5}+b_{33} a f_{3}+b_{33}\right) E^{*} S^{*}+b c_{2} b_{22} f_{2} f_{5} Q^{*} S^{*}+b_{11}\left(b_{22} b_{33}-c_{1}\right.$ $\left.d f_{4} f_{6} E^{*} Q^{*}\right), \varepsilon_{1}=b c_{2} f_{2} f_{5} Q^{*} S^{*}+\left(\beta_{1}+f_{1}\right)\left(a f_{3}+\beta_{1}\right) E^{*} S^{*}$ $+b_{11} b_{22}+b_{11} b_{33}+b_{22} b_{33}-c_{1} d f_{4} f_{6} E^{*} Q^{*}$, and $\varepsilon_{2}=b_{11}+$ $b_{22}+b_{33}$.

The system has the real part of eigenvalues as negative, if it satisfies $b_{11}>0, b_{22}>0, b_{33}>0$ and $b_{22} b_{33}>c_{1} d f_{4} f_{6} E^{*} Q^{*}$ i.e., $B<a f_{3} E^{*}+b f_{5} Q^{*}+\beta_{1} E^{*}+2 \mu S^{*}, \beta_{1} S^{*}+d f_{6} Q^{*}<\mu+$ $f_{1} S^{*}, \quad c_{1} f_{4} E^{*}+c_{2} f_{2} S^{*}<\mu, \quad\left(\beta_{1} S^{*}-f_{1} S^{*}+d f_{6} Q^{*}-\mu\right)$ $\left(c_{1} f_{4} E^{*}+c_{2} f_{2} S^{*}-\mu\right)>c_{1} d f_{4} f_{6} E^{*} Q^{*}$.

Hence, satisfying the Routh-Hurwitz criterion, the endemic is said to be locally asymptotically stable.

4.2. Global Stability. In this section, global stability for the endemic equilibrium point is studied using the Lyapunov function.

Theorem 3. Endemic point $E_{d}^{*}$ is asymptotically globally stable.
Proof. Let us assume the Lyapunov function $L^{*}$ as

$$
\begin{aligned}
& L^{*}(t)=\frac{1}{2}\left(S-S^{*}\right)^{2}+k_{1} \frac{1}{2}\left(E-E^{*}\right)^{2}+k_{2} \frac{1}{2}\left(Q-Q^{*}\right)^{2}, \\
& \frac{\mathrm{d} L^{*}}{\mathrm{~d} t}=\left(S-S^{*}\right)\left(B S-\beta_{1} S E-\frac{\beta_{2} S E}{a+S}-\frac{\beta_{4} S Q}{b+S}-\mu S^{2}\right) \\
& +k_{1}\left(E-E^{*}\right)\left(\beta_{1} S E+\frac{\beta_{2} S E}{a+S}-\frac{\beta_{3} E Q}{d+E}-\mu E\right) \\
& +k_{2}\left(Q-Q^{*}\right)\left(\frac{c_{1} \beta_{3} E Q}{d+E}+\frac{c_{2} \beta_{4} S Q}{b+S}-\mu Q\right) \\
& =\left(S-S^{*}\right)^{2}\left(B-\beta_{1} E-\frac{\beta_{2} E}{a+S}-\frac{\beta_{4} Q}{b+S}-\mu\left(S+S^{*}\right)\right) \\
& +k_{1}\left(E-E^{*}\right)^{2}\left(\beta_{1} S+\frac{\beta_{2} S}{a+S}-\frac{\beta_{3} Q}{d+E}-\mu\right) \\
& +k_{2}\left(Q-Q^{*}\right)^{2}\left(\frac{c_{1} \beta_{3} E}{d+E}+\frac{c_{2} \beta_{4} S}{b+S}-\mu\right) \\
& +S^{*}\left(S-S^{*}\right)\left(B-\mu S^{*}\right)-\frac{\beta_{2}}{a+S} \\
& \cdot\left(S^{*} E\left(S-S^{*}\right)-S E^{*}\left(E-E^{*}\right)\right. \\
& \left.-\frac{\beta_{4}}{b+S} \mid S^{*} Q\left(S-S^{*}\right)-k_{2} c_{2} S Q^{*}\left(Q-Q^{*}\right)\right) \\
& -\beta_{1}\left(S^{*} E\left(S-S^{*}\right)-k_{1} S E^{*}\left(E-E^{*}\right)\right) \\
& -\frac{\beta_{3}}{d+E}\left(k_{1} Q E^{*}\left(E-E^{*}\right)-k_{2} c_{1} Q^{*} E\left(Q-Q^{*}\right)\right) \\
& -\mu\left(k_{1} E^{*}\left(E-E^{*}\right)+k_{2} Q^{*}\left(Q-Q^{*}\right)\right) .
\end{aligned}
$$

where $\left(\mathrm{d} L^{*} / \mathrm{d} t\right)<0$ when $\beta_{1} S+\left(\beta_{2} S /(a+S)\right)<\left(\beta_{3} Q /\right.$ $(d+E))+\mu$ and $\left(c_{1} \beta_{3} E /(d+E)\right)+\left(c_{2} \beta_{4} S /(b+S)\right)<\mu$, $B<\mu S^{*}$. Hence, by LaSalle's invariance principle [16], the endemic equilibrium point is globally stable.

\section{Z-Control}

In this section, Z-control is applied on the abovementioned model. The basic idea of $\mathrm{Z}$-control approach is adopted from [17-20]. Z-control plays a vital role in reducing chaos and/or oscillation and thus in achieving the stability of the model. The Z-control approach is used to derive an expression for the system input say $u(t)$, so that the actual system output $y(t)$ will be forced to achieve the desired output $y_{d}(t)$. Moreover, Z-control is more useful if the difference between the actual output of the system and its desired output is negligible, namely, if $e(t)=y(t)-y_{d}(t) \longrightarrow 0$ as $t \longrightarrow \infty$. This objective is achieved by forcing error function $e(t)$ to 
converge exponentially to zero when it satisfies the following equation:

$$
\dot{e}(t)=-\lambda e(t), \quad \text { for } \lambda>0 .
$$

This equation is also known as the design formula and $\lambda$ is the design parameter which is strictly positive indicating the convergence rate. The $\mathrm{Z}$-control approach is based on the following steps. Firstly, define the error function and, secondly, use the design formula whenever required to obtain the explicit expression of $u(t)$.

Now, by applying indirect Z-controller to the quarantine population, system (1) is rewritten as

$$
\begin{aligned}
& \frac{\mathrm{d} S}{\mathrm{~d} t}=B S-\beta_{1} S E-\frac{\beta_{2} S E}{a+S}-\frac{\beta_{4} S Q}{b+S}-\mu S^{2}, \\
& \frac{\mathrm{d} E}{\mathrm{~d} t}=\beta_{1} S E+\frac{\beta_{2} S E}{a+S}-\frac{\beta_{3} E Q}{d+E}-\mu E, \\
& \frac{\mathrm{d} Q}{\mathrm{~d} t}=\frac{c_{1} \beta_{3} E Q}{d+E}+\frac{c_{2} \beta_{4} S Q}{b+S}-\mu Q-u(t) Q
\end{aligned}
$$

$$
\begin{aligned}
\dot{e}_{2}(t)= & -\lambda e_{2}(t), \\
\Longrightarrow & \left(\ddot{E}(t)-\ddot{E}_{d}(t)\right)+\lambda\left(\dot{E}(t) t-n \dot{E}_{d} q(t)\right)=-\lambda\left(\left(\dot{E}(t)-\dot{E}_{d}(t)\right)+\lambda\left(E(t)-E_{d}(t)\right)\right) . \\
\Longrightarrow & \beta_{1} \dot{S} E+\beta_{1} S \dot{E}+\frac{\beta_{2} S \dot{E}}{a+S}+\frac{\beta_{2} \dot{S} E}{a+S}-\frac{\beta_{2} \dot{S} S \dot{E}}{(a+S)^{2}}-\frac{\beta_{3} E \dot{Q}}{d+E}-\frac{\beta_{3} \dot{E} Q}{d+E}+\frac{\beta_{3} E \dot{E} Q}{(d+E)^{2}}-\mu \dot{E}-\ddot{E}_{d}(t) \\
& +\lambda\left(\dot{E}(t)-\dot{E}_{d}(t)\right)=-\lambda\left(\left(\dot{E}(t)-\dot{E}_{d}(t)\right)+\lambda\left(E(t)-E_{d}(t)\right)\right) . \\
\Longrightarrow & \dot{Q}=\frac{(d+E)}{\beta_{3} E}\left[\beta_{1} \dot{S} E+\beta_{1} S \dot{E}+\frac{\beta_{2} S \dot{E}}{a+S}+\frac{\beta_{2} \dot{S} E}{a+S}-\frac{\beta_{2} \dot{S} S \dot{E}}{(a+S)^{2}}-\frac{\beta_{3} \dot{E} Q}{d+E}+\frac{\beta_{3} E \dot{E} Q}{(d+E)^{2}}-\mu \dot{E}-\ddot{E}_{d}(t)\right. \\
& \left.+\lambda\left(\dot{E}(t)-\dot{E}_{d}(t)\right)+\lambda\left(\left(\dot{E}(t)-\dot{E}_{d}(t)\right)+\lambda\left(E(t)-E_{d}(t)\right)\right)\right] .
\end{aligned}
$$

We finally obtain the expression for Z-controller as

$$
\begin{aligned}
\Rightarrow & u(t)=\frac{c_{1} \beta_{3} E}{d+E}+\frac{c_{2} \beta_{4} S}{b+S}-\mu-\frac{(d+E)}{\beta_{3} E Q}\left[\beta_{1} \dot{S} E+\beta_{1} S \dot{E}+\frac{\beta_{2} S \dot{E}}{a+S}+\frac{\beta_{2} \dot{S} E}{a+S}-\frac{\beta_{2} \dot{S} S \dot{E}}{(a+S)^{2}}-\frac{\beta_{3} \dot{E} Q}{d+E}+\right. \\
& \left.\frac{\beta_{3} E \dot{E} Q}{(d+E)^{2}}-\mu \dot{E}-\ddot{E}_{d}(t)+\lambda\left(\dot{E}(t)-\dot{E}_{d}(t)\right)+\lambda\left(\left(\dot{E}(t)-\dot{E}_{d}(t)\right)+\lambda\left(E(t)-E_{d}(t)\right)\right)\right] .
\end{aligned}
$$

Theorem 4. Starting from the positive initial state $[S(0), E(0), Q(0)]^{T}$, the error function $e_{1}$ of model (12) equipped with Z-controller (16) converges to zero exponentially for a continuously differentiable and bounded desired state $E_{d}(t)$.

Proof. According to (16), we consider $e_{2}=\dot{e}_{1}+\lambda e_{1}$, and substituting it in $\dot{e}_{2}(t)=-\lambda e_{2}(t)$, we get

$$
\ddot{e}_{1}+2 \lambda \dot{e}_{1}+\lambda^{2} e_{1}=0 .
$$

But,

The solution of this second-order differential equation is calculated as

$$
e_{1}=\left(a_{1}+a_{2} t\right) \exp (-\lambda t), \quad \forall t \geq 0
$$

where $a_{1}$ and $a_{2}$ are arbitrary constants. Using the initial value condition, we obtain $a_{1}=E(0)-E_{d}(0), a_{2}=\dot{E}$ $(0)-\dot{E}_{d}(0)+\lambda\left(E(0)-E_{d}(0)\right)$. 


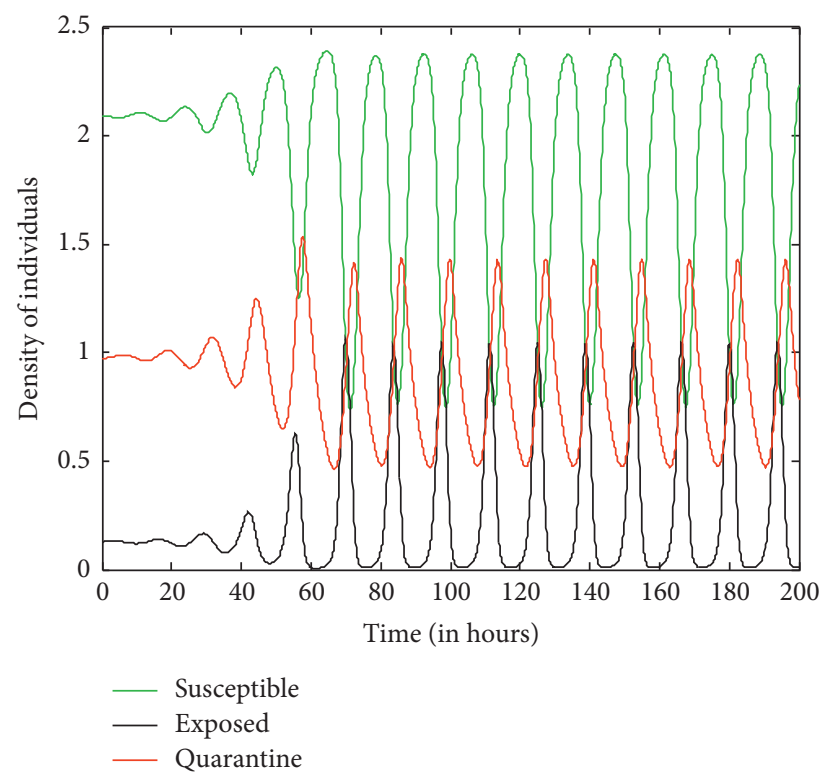

FIgURE 2: Transmission of COVID-19 in the diabetic/hypertension population.

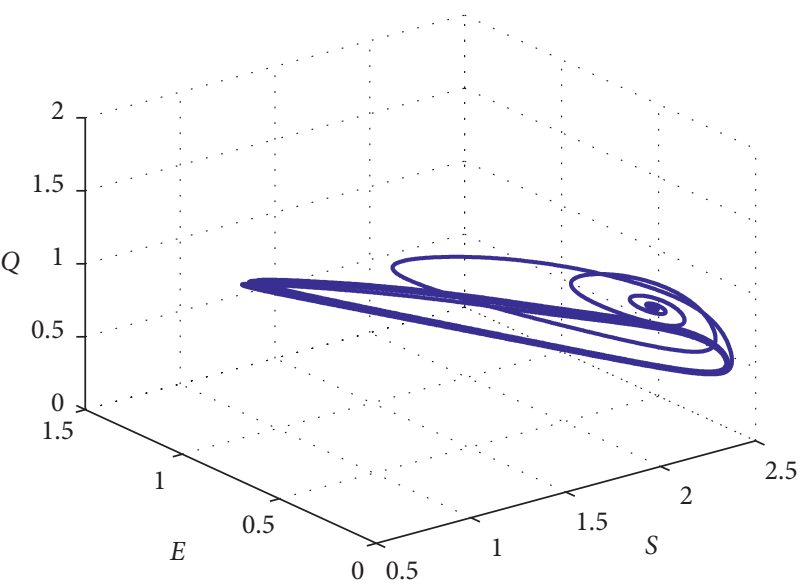

FIgURE 3: Scatter diagram.

According to Lemma 1 in [21], $\exists \bar{c}>0$ and $\bar{\lambda}>0$ such that $e_{1} \leq \bar{c} \exp (-\lambda t)$. Thus, the tracking error exponentially converges to zero for $\bar{\lambda}>0$, which implies that the population exposed to COVID-19 converges exponentially to its desired state.

\section{Numerical Simulation}

In this section, simulation is developed to study the importance of quarantine for the population suffering from diabetes or hypertension in the COVID-19 environment. Data given in Table 1 have been used for the numerical simulation.

Figure 2 shows the plotting of susceptible class, exposed class, and quarantine class. It depicts the model completely indicating the movement of individuals from one compartment to another compartment.
Figure 3 is plotted with respect to all the compartments, namely, susceptible, exposed, and quarantine at the endemic equilibrium. It shows the periodic nature of system (1).

Figure 4 shows that the individuals exposed to COVID19 are quarantining themselves or are maintaining social distance to avoid the transmission of the disease.

Figure 5 shows the cycle's oscillation for the endemic equilibrium point at $\beta_{3}=1.8$ without applying Z-controller. With further increase in $\beta_{3}$, the system becomes more chaotic in nature showing its instability. For better understanding of the periodic oscillation and chaotic nature, we draw a bifurcation diagram taking $\beta_{3}$ as the bifurcating parameter.

Figure 6 shows the periodicity along each compartment with respect to $\beta_{3}$. Here, the maximum and the minimum values are plotted with blue and red, respectively. Here, it is observed that if the population is exposed to COVID-19, 


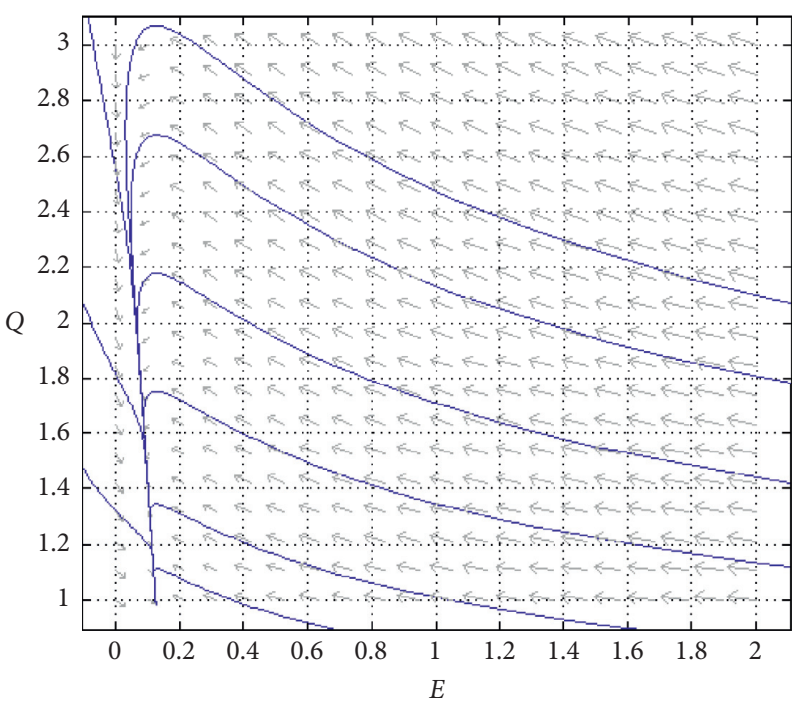

FIgURE 4: Behaviour of population exposed to COVID-19.

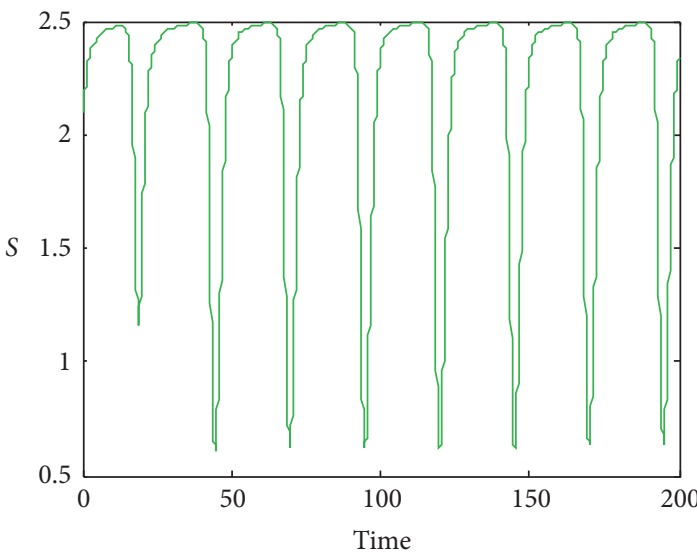

(a)

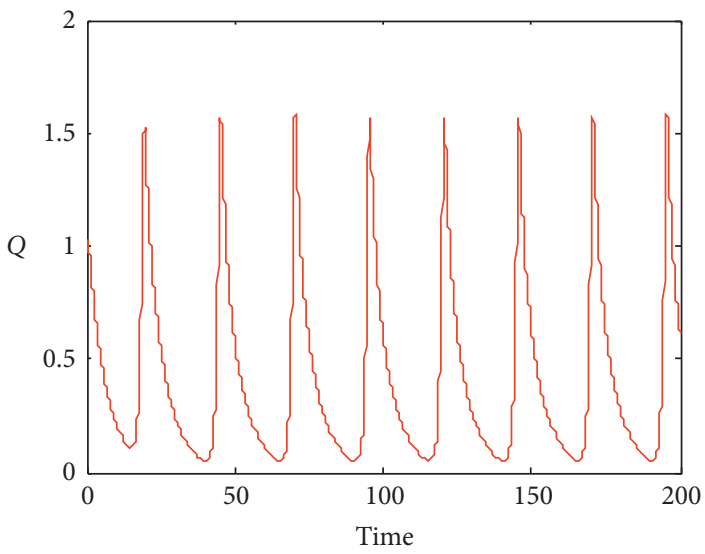

(c)

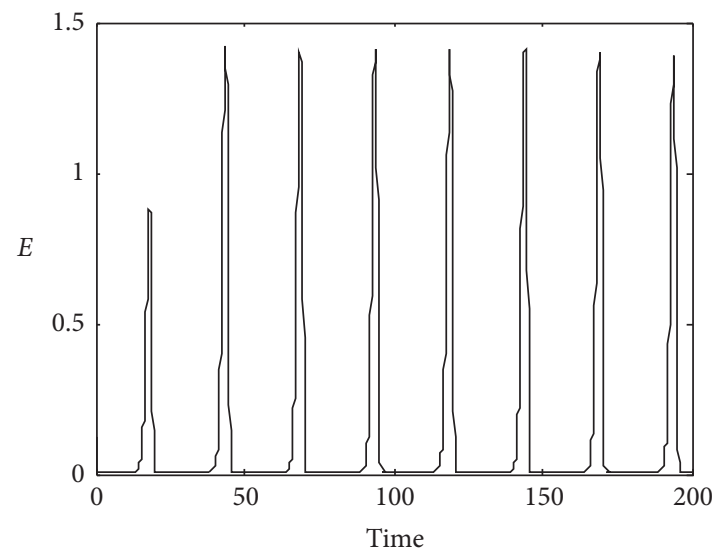

(b)

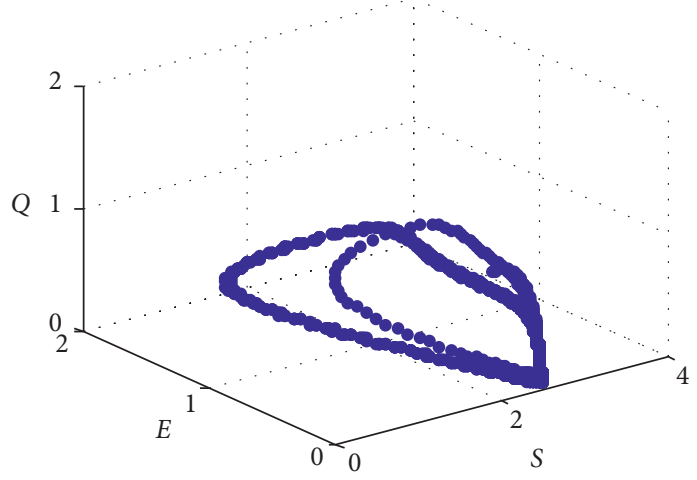

(d)

Figure 5: Oscillations at $\beta_{3}=1.8$.

more and more individuals undergo quarantine increasing the periodicity, which further turns into chaos. In order to make the system stable and free from chaos or periodicity, we apply Z-control to system (1).
By implementing Z-control as explained in Section 5 to system (1), one can observe from Figure 7 that the system has been made free from chaos or periodicity, making the system stable. Here, $E_{d}=0.001$, i.e., the desired value of $E_{d}$. We can 


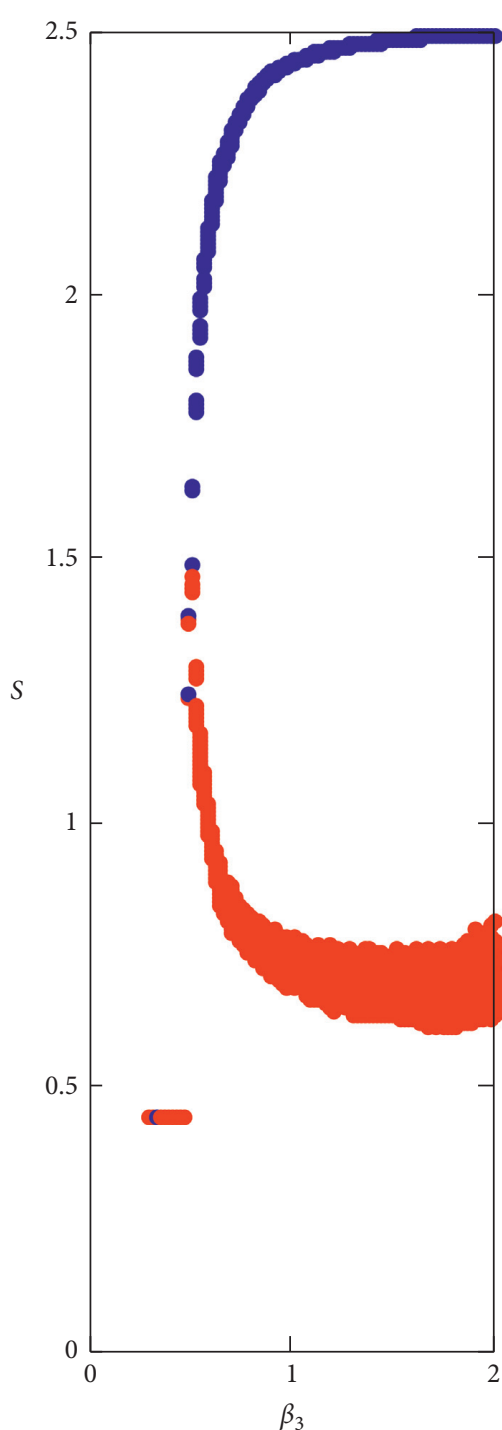

(a)

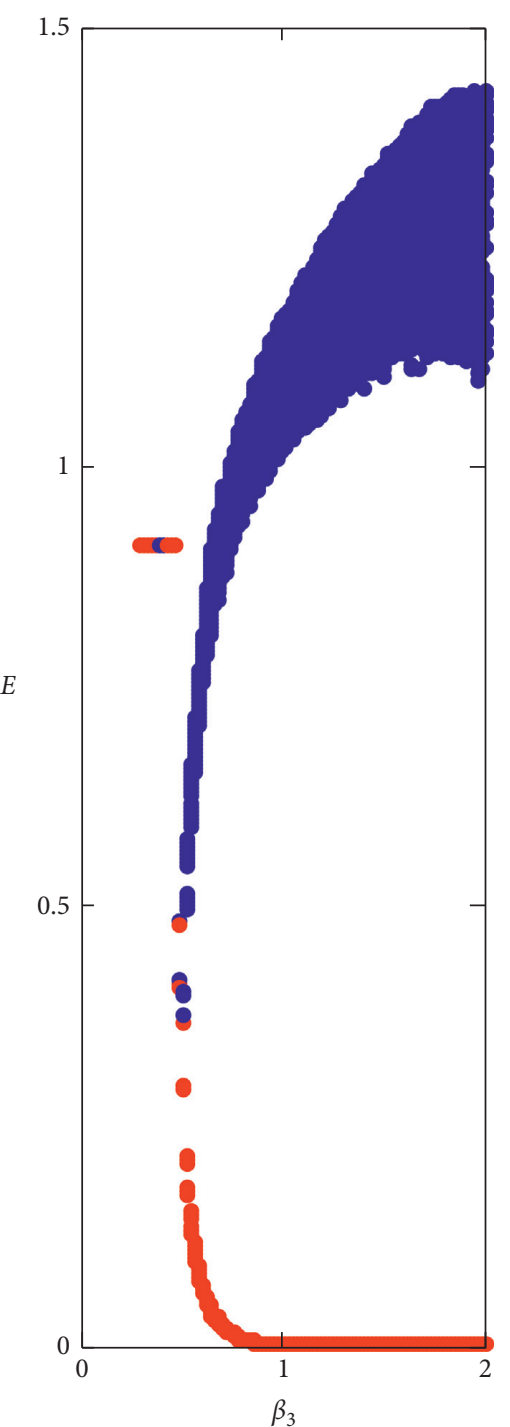

(b)

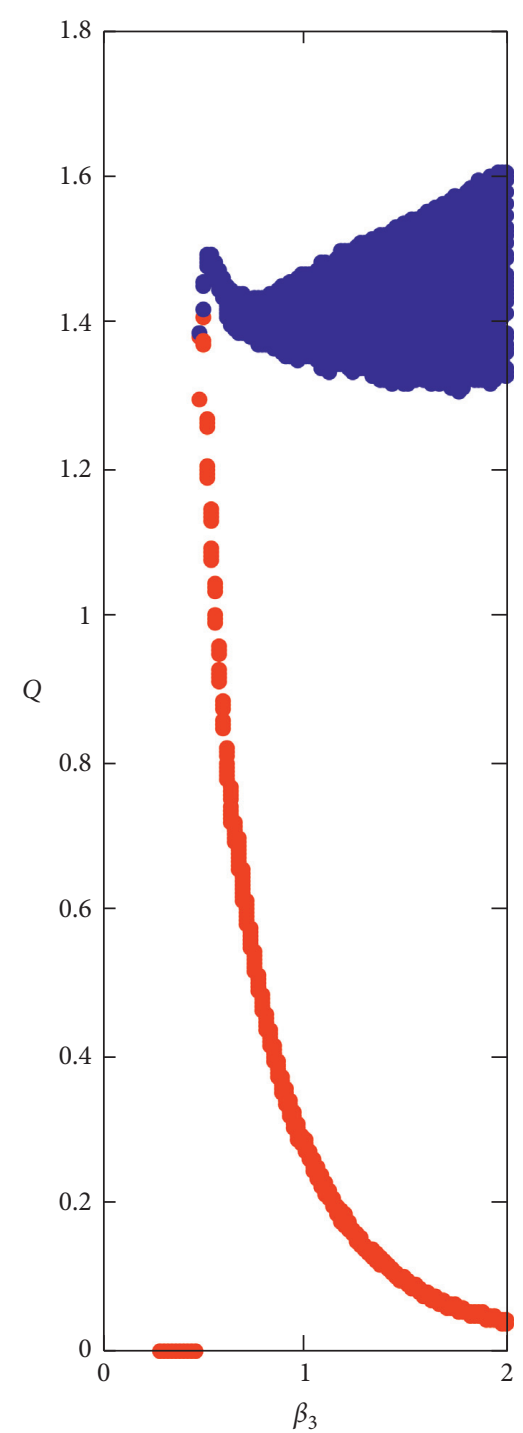

(c)

Figure 6: Bifurcation diagram.

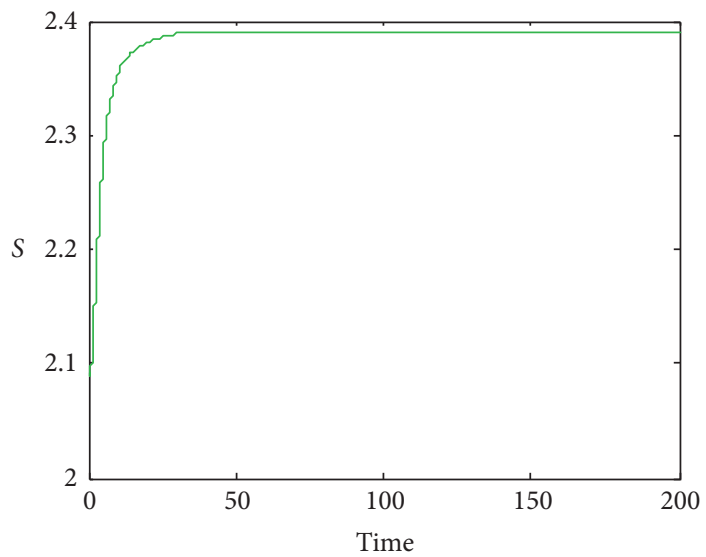

(a)

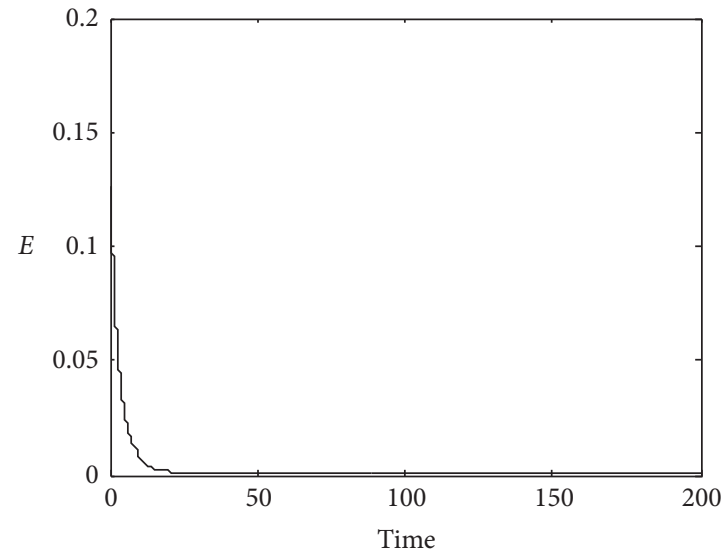

(b)

Figure 7: Continued. 


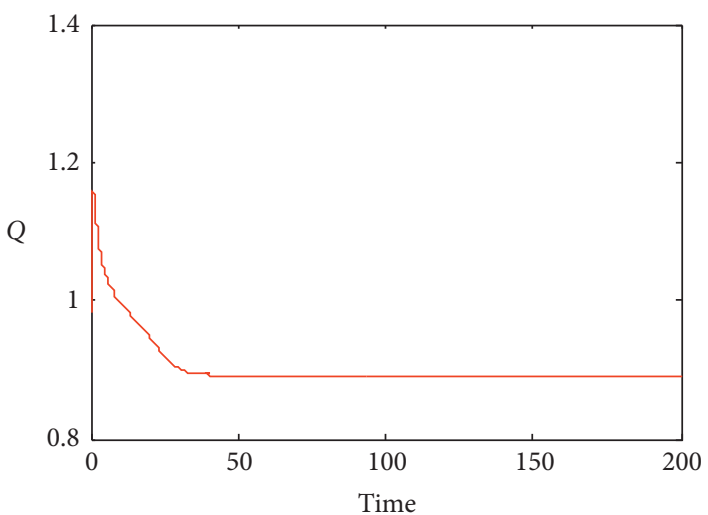

(c)

Figure 7: Implementation of Z-control.

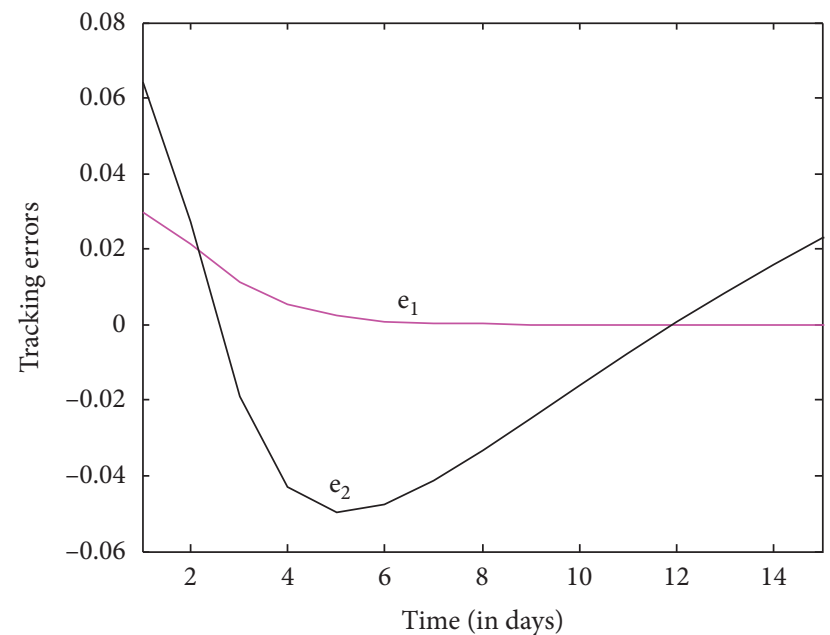

Figure 8: Error function.

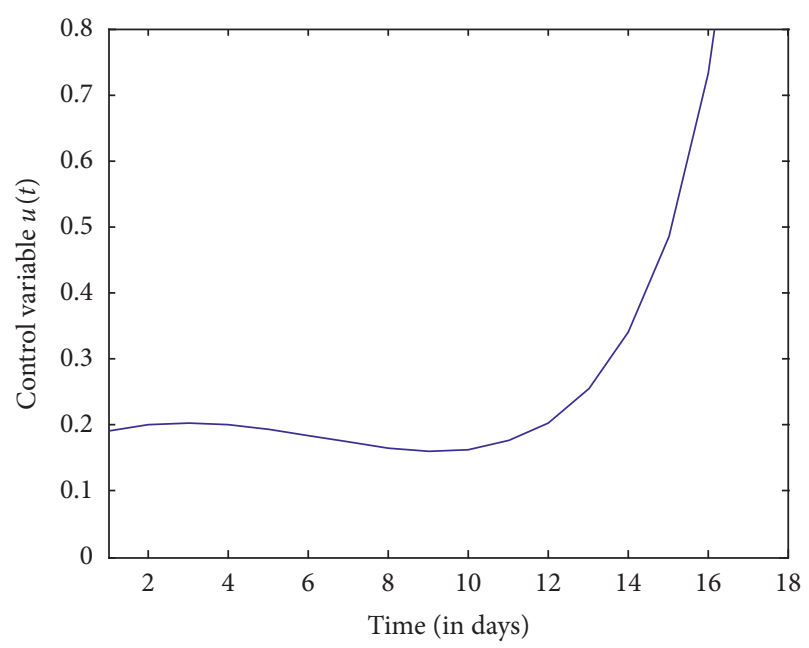

FIgURE 9: Control variable. see that Figure 5 gives us the uncontrolled system with unstable dynamics and Figure 7 gives us the controlled system with stable dynamics. Figure 7 is interpreted as the susceptible individuals, before exposure to COVID-19, quarantine or isolate themselves to avoid contact with the disease.

Figure 8 shows the tracking errors which have been used in the design formula. Figure 9 depicts the behaviour of the control variable applied in the Z-control model. It shows that initially $20 \%$ control is required and later on, as the exposure to disease further increases, control to be applied also increases, i.e., a greater number of individuals are to be quarantined.

\section{Conclusion}

This paper considers a mathematical model for the population suffering from diabetes or hypertension exposed to COVID-19. Here, we have calculated the reproduction number when there is no exposure to COVID19 which turns out to be 2.66 , indicating that a single individual asks atleast two individuals to go for quarantine. In a similar manner, the reproduction number in the endemic stage is calculated as 5.33, indicating that at least 5 individuals quarantine themselves when they are exposed to the disease. Next, we have derived the conditions for the system to be locally stable with the help of the Routh-Hurwitz criterion. Global stability is also studied for the endemic equilibrium point. In this paper, we have investigated $\mathrm{Z}$-control in order to control the disease dynamics. Here, the control is applied to the quarantine class in order to obtain the desired exposure in attaining the stability of the system. At the end, the numerical simulation is established indicating the importance of quarantine which helps reduce the chaos of the system. If individuals quarantine themselves at the onset of COVID19 , it keeps them safe, which is the only way to protect oneself as there is no vaccine till date. 


\section{Data Availability}

The data used to support the findings of this study are included within the article.

\section{Conflicts of Interest}

The authors declare that there are no conflicts of interest regarding the publication of this paper.

\section{Acknowledgments}

The authors thank DST-FIST for the technical support to the department with the file \# MSI-097. ENJ was funded by UGC: National Fellowship for Other Backward Classes (NFO-2018-19-OBC-GUJ-71790).

\section{References}

[1] https://www.worldometers.info/coronavirus/\#countries.

[2] https://www.who.int/health-topics/coronavirus\#tab=tab_1.

[3] Q. Li, X. Guan, P. Wu et al., "Early transmission dynamics in Wuhan, China, of novel coronavirus-infected pneumonia," New England Journal of Medicine, vol. 382, no. 13, 2020.

[4] M. Batista, "Estimation of the final size of the COVID-19 epidemic," MedRxiv, vol. 10, no. 2, 2020.

[5] L. Peng, W. Yang, D. Zhang, C. Zhuge, and L. Hong, "Epidemic analysis of COVID-19 in China by dynamical modeling," 2020, https://arxiv.org/abs/2002.06563.

[6] P. Sun, X. Lu, C. Xu, W. Sun, and B. Pan, "Understanding of COVID-19 based on current evidence," Journal of Medical Virology, vol. 92, no. 6, pp. 548-551, 2020.

[7] J. F. Rabajante, "Insights from early mathematical models of 2019-nCoV acute respiratory disease (COVID-19) dynamics," 2020, https://arxiv.org/abs/2002.05296.

[8] M. Chinazzi, J. T. Davis, M. Ajelli et al., "The effect of travel restrictions on the spread of the 2019 novel coronavirus (COVID-19) outbreak," Science, vol. 368, no. 6489, pp. 395400, 2020.

[9] A. J. Kucharski, T. W. Russell, C. Diamond et al., "Early dynamics of transmission and control of COVID-19: a mathematical modelling study," The Lancet Infectious Diseases, vol. 20, pp. 553-558, 2020.

[10] B. Tang, N. L. Bragazzi, Q. Li, S. Tang, Y. Xiao, and J. Wu, "An updated estimation of the risk of transmission of the novel coronavirus (2019-nCov)," Infectious Disease Modelling, vol. 5, pp. 248-255, 2020.

[11] B. Tang, X. Wang, Q. Li et al., "Estimation of the transmission risk of the 2019-nCoV and its implication for public health interventions," Journal of Clinical Medicine, vol. 9, no. 2, pp. 462-474, 2020.

[12] A. K. Alzahrani, A. S. Alshomrani, N. Pal, and S. Samanta, "Study of an eco-epidemiological model with Z-type control," Chaos, Solitons \& Fractals, vol. 113, pp. 197-208, 2018.

[13] S. Samanta, "Study of an epidemic model with Z-type control," International Journal of Biomathematics, vol. 11, no. 7, Article ID 1850084, 2018.

[14] O. Diekmann, J. A. P. Heesterbeek, and J. A. Metz, "On the definition and the computation of the basic reproduction ratio R0 in models for infectious diseases in heterogeneous populations," Journal of Mathematical Biology, vol. 28, no. 4, pp. 365-382, 1990.
[15] E. J. Routh, A Treatise on the Stability of a Given State of Motion: Particularly Steady Motion, Macmillan and Company, New York, NY, USA, 1877.

[16] J. P. LaSalle, The Stability of Dynamical Systems, Society for Industrial and Applied Mathematics, Philadelphia, PA, USA, 1976.

[17] D. Guo and Y. Zhang, "Neural dynamics and Newton-Raphson iteration for nonlinear optimization," Journal of Computational and Nonlinear Dynamics, vol. 9, no. 2, 2014.

[18] Y. Zhang, X. Yan, B. Liao, Y. Zhang, and Y. Ding, "Z-type control of populations for Lotka-Volterra model with exponential convergence," Mathematical Biosciences, vol. 272, pp. 15-23, 2016.

[19] D. Lacitignola, F. Diele, C. Marangi, and A. Provenzale, "On the dynamics of a generalized predator-prey system with Ztype control," Mathematical Biosciences, vol. 280, pp. 10-23, 2016.

[20] B. Liao and Y. Zhang, "Different complex ZFs leading to different complex ZNN models for time-varying complex generalized inverse matrices," IEEE Transactions on Neural Networks and Learning Systems, vol. 25, no. 9, pp. 1621-1631, 2013.

[21] Z. Zhang and Y. Zhang, "Design and experimentation of acceleration-level drift-free scheme aided by two recurrent neural networks," IET Control Theory \& Applications, vol. 7, no. 1, pp. 25-42, 2013. 